\title{
Impact of Pelvic Pain and Endometriosis on Patient-Reported Outcomes and Experiences of Benign Hysterectomy: A Study from the Swedish National Register for Gynecological Surgery
}

Hanna Grundström, Siw Alehagen, Carina Berterö and Preben Kjölhede

The self-archived postprint version of this journal article is available at Linköping University Institutional Repository (DiVA):

http:// urn.kb.se/ resolve?urn=urn:nbn:se:liu:diva-143941

N.B.: When citing this work, cite the original publication.

Grundström, H., Alehagen, S., Berterö, C., Kjölhede, P., (2017), Impact of Pelvic Pain and

Endometriosis on Patient-Reported Outcomes and Experiences of Benign Hysterectomy: A Study from the Swedish National Register for Gynecological Surgery, J ournal of Women's Health.

https:// doi.org/ 10.1089/jwh.2017.6546

Original publication available at:

https:// doi.org/10.1089/jwh.2017.6546

Copyright: Mary Ann Liebert

http:// www.liebertpub.com/ 
An original research article

entitled

Impact of pelvic pain and endometriosis on patient-reported outcomes and experiences of benign hysterectomy: A register study from the Swedish National Register for Gynecological Surgery.

by

Hanna Grundström, $\mathrm{RNM}^{1,2}$, Siw Alehagen, RNM, $\mathrm{PhD}^{2}$, Carina Berterö, RNT, BSc, MScN, $\mathrm{PhD}^{2}$, and Preben Kjølhede, $\mathrm{MD}, \mathrm{PhD}^{3,4}$

\section{Affiliations:}

${ }^{1}$ Department of Obstetrics and Gynecology, Vrinnevi Hospital, SE- 60182 Norrköping, Sweden

${ }^{2}$ Department of Medical and Health Sciences, Faculty of Medicine and Health Science, Linköping University, SE- 58183 Linköping, Sweden

${ }^{3}$ Department of Obstetrics and Gynecology, and ${ }^{4}$ Department of Clinical and Experimental Medicine, Faculty of Medicine and Health Science, Linköping University, SE- 58183

Linköping, Sweden

\section{Corresponding author}

Hanna Grundström, RNM

Department of Obstetrics and Gynecology

Vrinnevi Hospital

SE - 60182 Norrköping, Sweden

E-mail: hanna.grundstrom@liu.se

Phone: +46709758183

Running title: PREMs and PROMs after benign hysterectomy 


\section{Abstract}

Background: The study objective was to analyze and compare patient-reported experience measures (PREMs) and patient-reported outcome measures (PROMs) after hysterectomy in women with and without a preoperative complaint of pelvic pain associated with and without a confirmed diagnosis of endometriosis.

Methods Retrospective nationwide register study. Data on 28776 hysterectomies performed on benign indication between 2004 and 2016 were retrieved from the Swedish National Register for Gynecological Surgery. Multivariable logistic regression models were used to compare the PREMs and PROMs items. The results are presented as adjusted odd ratios (aORs) and 95\% confidence intervals (CI).

Results: Regardless of the occurrence of pelvic pain preoperatively and a diagnosis of endometriosis, one year after surgery the women were satisfied or very satisfied ( $>90 \%)$ with the hysterectomy, and their medical condition was improved or much improved (>95\%). The women with a preoperative complaint of pelvic pain and endometriosis more often reported excessively short hospital stays (aOR 1.45, 95\%CI; 1.17-1.79), more severe complications after discharge (aOR 2.02, 95\%CI; 1.59-2.66) at the eight-week follow-up and at the one-year follow-up (aOR 2.31, 95\%CI; 1.57-3.39), and more dissatisfaction with the operation (aOR 1.83, 95\%CI; 1.35-2.48) than preoperative pelvic pain-free women without endometriosis at the one-year follow-up.

Conclusions: The majority of the women were satisfied after their hysterectomy. The women with pelvic pain and endometriosis were at a higher risk of being dissatisfied. Pelvic pain per se seemed to be the main factor affecting the rating in the PREMs and PROMs, and the endometriosis was a significant contributing factor.

Key words: Endometriosis, Experience, Hysterectomy, Outcome, Pelvic pain, 


\section{Introduction}

Endometriosis is characterized by the growth of endometrial-like cells, stroma and glands outside the uterine cavity, which induces a chronic inflammatory reaction. The etiology of endometriosis is still in dispute; immune, hormonal, genetic, and epigenetic factors may all be involved. Several theories on the pathogenesis of endometriosis have been proposed $^{1}$. New research has shown that dysregulation of the signaling pathways, for instance the Wnt, $\beta$-catenin and the HOX signaling pathways resulting in altered gene expression, cytokine generation, and proliferation, are involved in the development of endometriosis. This causes an alteration in the cellular function and consequently implies a genetic disorder as the etiology of endometriosis ${ }^{2-4}$.

The endometrial-like tissue and inflammation growing in aberrant locations may cause chronic pelvic pain (CPP), dysmenorrhea, dyspareunia or infertility. In addition, intestinal and/or urinary complaints are common ${ }^{5,6}$. The ectopic tissues are not necessarily the source of the pain symptoms. Around $25 \%$ of women diagnosed with endometriosis are asymptomatic ${ }^{7}$. Therefore, the diagnosis and treatment of endometriosis and CPP is complex

8. The treatment involves medical and surgical interventions, with hysterectomy being one of the ultimate treatment modalities. It is estimated that endometriosis is the indication for 2$12 \%$ of all hysterectomies ${ }^{9-12}$.

While women having hysterectomy on benign, non-painful gynecological disorders have excellent outcomes as regards cure of the symptoms or the disease, the role of hysterectomy in the treatment of CPP is controversial and its effect is dubious ${ }^{13}$. The outcomes of hysterectomy among women with CPP have been the subject of a limited number of studies. Pain is reported to persist in approximate $25 \%$ of subjects ${ }^{14}$, and in up to $40 \%$ in specific subgroups of women ${ }^{15}$. A Danish study found risk factors for CPP after hysterectomy to be preoperative pelvic pain, pain as the indication for surgery, and having 
pain problems elsewhere ${ }^{16}$. In other studies, additional risk factors for postoperative pain have been reported such as symptomatic endometriosis ${ }^{17}$ and depression ${ }^{18}$.

Many women with endometriosis and CPP are convinced that a hysterectomy may relieve the pain and they consider the procedure to be "the last resort" - a potential permanent solution to their pain, and have expectations of being pain-free afterwards ${ }^{19}$. Given this background, the effectiveness of hysterectomy must justify the operation, ensuring that it generates a subjective positive effect.

One way to evaluate the effectiveness of a treatment and satisfaction with it is to use patient-reported experience measures (PREMs) and patient-reported outcome measures (PROMs). PREMs provide an understanding of patients' views on their experience while receiving care, while PROMs are used in the search for patients' views on their health or the intervention being studied. The use of information on both patient experience and outcomes generates a broad understanding of the quality of the care from the patients' point of view ${ }^{20}$.

We conducted this study with the aim of determining whether the PREMs and PROMs after benign hysterectomy differed in women with and without a confirmed diagnosis of endometriosis associated with or without a preoperative complaint of pelvic pain.

We hypothesized-dthat women with a confirmed diagnosis of endometriosis at the hysterectomy score lower on PREMs and PROMs than women who have hysterectomy for other benign gynecological conditions. The occurrence of a concurrent preoperative complaint of pelvic pain will influence these outcomes even more negatively. 


\section{Materials and methods}

This retrospective study is based on data from the Swedish National Register for Gynecological Surgery (GynOp). The register was established in 1994 and data on hysterectomies have been collected since $1997^{21}$. The data were retrieved from 57 hospitals enrolling patients in the register, including more than $75 \%$ of all benign hysterectomies performed in Sweden during this period ${ }^{21}$.

All women undergoing gynecological surgery receive written information about the register prior to surgery, and have the opportunity to decline participation or withdraw consent later on. The written information announces that the collected data may be used for research purposes. In accordance with the Swedish legislation, the patient gives formal consent to participating in the register by answering the health declaration and the questionnaires.

Data are collected prospectively using patient questionnaires and doctors’ records. Preoperatively, the patient completes a health declaration and answers questions on subjective symptoms. Eight weeks and one year postoperatively, a questionnaire is sent to the patient with questions regarding health status, recovery and the experiences and outcomes of the surgery.

The surgeon registers data in a computerized preoperative form, an operation form and a discharge form. The surgeon evaluates the postoperative patient questionnaires regarding complications and the severity of the complications ${ }^{21}$.

In this study, the extracted data included 1) patient demographics from the health declaration: age, parity, height and weight enabling the calculation of body mass index (BMI), smoking habits and employment, 2) clinical data accumulated peri- and postoperatively: American Society of Anesthesiologists classification, mode of hysterectomy (abdominal, vaginal or laparoscopy), hysterectomy type (total or subtotal), whether the patient had a 
remaining ovary at the conclusion of the surgery, and complications during the hospital stay,

3) PREMs and PROMs from a) the eight-week questionnaire comprising questions concerning patients' experiences of length of hospital stay, occurrence of complications after discharge, and current medical condition, b) the one-year questionnaire covering the questions about satisfaction with the operation, occurrence of complications after discharge, and current medical condition. The questions and the options for possible answers in brackets related to the PREMs and PROMs in the eight-week questionnaire were formulated as follows: "How do you rate the length of your hospital stay?” (Too long/Adequate/Too short) "Have you experienced any unexpected complications related to the surgery?" (No/Yes, mild/Yes, severe/Yes, both mild and severe) "How do you rate the results of the surgery so far? My medical condition is:" (Much improved/Improved/Unchanged/Worse/Much worse). The PREM and PROM questions and options for possible answers in the one-year questionnaire were formulated as: “Regarding the results of the operation, I am:” (Very satisfied/Satisfied/Neither-nor/Dissatisfied/Very dissatisfied) "During the period from two months after the surgery until this day have you had any complications related to the surgery?” (No/Yes, mild/Yes, severe). "My medical condition is:” (Much improved/Improved/Unchanged/Worse/Much worse). The question concerning the result of the surgery in the one-year questionnaire was included in 2010. The women who underwent surgery less than one year prior to the data retrieval did not have the possibility to answer the one-year questionnaires and therefore only contributed to the eight-week analysis.

All women who were registered in the GynOp as having undergone hysterectomy on benign indication between January 01, 2004 and July 31, 2016 were eligible for the study. Exclusion criteria were age $>55$ years; having the hysterectomy on prolapse or incontinence indications; undergoing hysterectomy for complications related to pregnancy or post-partum complications; having any suspected malignancy, 
endometrial or cervical dysplasia or having a prophylactic hysterectomy due to heredity for gynecological malignancy. Women with no gynecological indication for the hysterectomy or for whom no indication was reported were also excluded.

Women with a subjective complaint of pain and an endometriosis diagnosis confirmed histopathologically were categorized as the index group (A). The three comparison groups consisted of (B) women with a subjective complaint of pelvic pain preoperatively with other diagnoses (such as menstrual bleeding disorder, uterine fibroids, ovarian cysts etc.) and no histopathologically verified endometriosis discovered during the operation; (C) women, preoperatively subjective pain-free, with a histopathologically verified endometriosis diagnosis discovered during the operation; and (D) women, preoperatively subjective painfree, with other diagnoses than endometriosis (such as menstrual bleeding disorder, uterine fibroids, ovarian cysts, etc.).

The study conforms to the STROBE Statement. The Regional Ethics Board of Linköping University (Reg. no. M19-07; amendment 2016/66-32) approved the study. Statistics

Statistical analyses were conducted with the software Statistica v 13.0 (Dell Software, 5 Polaris Way, Aliso Viejo, CA 92656, USA). Variables on continuous scales are described as mean, standard deviation and range. Nominal data are presented as frequency and percentage. In order to assess the outcome measures, multivariate logistic regression analyses were conducted. In the logistic regression analyses the outcome measures were dichotomized (Table 3). In the multivariate logistic regression models we adjusted the associations for known or potential confounding factors. The results of the outcome measures are given as crude and adjusted odds ratios (ORs or aORs) and 95\% confidence intervals (95\% CIs). In the multivariate logistic regression models adjustments were made simultaneously for known or presumed confounding factors for the outcome measurements, that is age, parity, BMI 
(continuous variable), American Society of Anesthesiologists' classification, smoking habits, employment, mode of hysterectomy, hysterectomy type, occurrence of bilateral oophorectomy, mode of anesthesia and occurrence of complications during hospital stay. 


\section{Results}

The selection of the study population is presented in the flow chart in Figure 1. As shown in Table 1 the women in the index group (A) were slightly younger, were more often overweight/obese, were more often smokers, had more comorbidity as indicated by the American Society of Anesthesiologists' score classification, and were less likely to have fulltime employment than the comparison groups (B to D). Concerning the clinical data, the women in the index group were the most likely to end up having a bilateral oophorectomy at the hysterectomy and to more often have complications during the hospital stay (Table 2).

The answers to the PREM and PROM questions obtained from the eight-week and the one-year questionnaires and the dichotomized transformation of the answers to the questions are presented in Table 3. The response rates to the eight-week and one-year questionnaires were $88.0 \%$ and $83.2 \%$, respectively. In general, all presented questions showed very high frequencies of "positive” estimations of the hysterectomy in all groups.

In the comparison of the PREMs and PROMs of the index group with those of the three comparison groups from the eight-week and the one-year questionnaire, many of the outcomes had statistically significant differences in the univariate analyses, as shown by the crude ORs in Table 4. When adjusting for the confounding factors in the multivariate logistic regression models, the assessment of the women with pelvic pain and with and without endometriosis (Group A vs. Group B) revealed that none of the outcomes from the eight-week questionnaire remained statistically significant. In contrast, among the women with a preoperative complaint of pelvic pain, both the dissatisfaction with the operation and experienced complications after the surgery at the one-year assessment remained significant, revealing higher odds ratios for dissatisfaction (aOR 1.30, 95\%CI; 1.02-1.65) and more experiences of severe complications (aOR 1.37, 95\%CI; 1.03-1.82) in women with endometriosis. Likewise, when analyzing the women with endometriosis with and without a 
preoperative complaint of pelvic pain (Group A vs. Group C) with multivariate logistic regression models, the group with a preoperative complaint of pelvic pain showed a higher risk of being dissatisfied with the result of the operation (aOR 1.58, 95\%CI; 1.02-2.45) than the pelvic pain-free group.

When comparing the women with a preoperative complaint of pelvic pain and endometriosis (Group A) with the preoperative pelvic pain-free women without endometriosis (Group D), the adjusted ORs in the multivariate logistic regression models remained statistically significant both at the eight-week assessment and at the one-year assessment. The women with preoperative complaint of pelvic pain and endometriosis (Group A) more often reported that the hospital stay was too short (aOR 1.45, 95\%CI; 1.17-1.79), experienced more severe complications after discharge (aOR 2.02, 95\%CI; 1.59-2.66 at eight weeks and aOR 2.31, 95\%CI; 1.57-3.39 at one year, respectively), and were more dissatisfied with the operation (aOR 1.83, 95\%CI; 1.35-2.48) than the preoperative pelvic pain-free women without endometriosis (Group D).

Interestingly, in the multivariate logistic regression models of the eight-week and the one-year assessments, the rating of the women's medical condition did not differ significantly between the index group (Group A) and the comparison groups (Group B-D) (Table 4). More than $95 \%$ of women in all groups claimed their medical condition was improved or much improved (Table 3). 


\section{Discussion}

This study revealed that women with pelvic pain and endometriosis who undergo hysterectomy, within one year of the surgery in general are satisfied or very satisfied with the operation and to a very high degree rate their medical condition as improved or much improved. Although there were no observed significant associations in the rating of the medical condition between the groups, women with pelvic pain and endometriosis had higher odds of being dissatisfied with the result of the operation. They also more often experienced severe complications after discharge than women with pelvic pain without endometriosis or women without pelvic pain with or without endometriosis. To the best of our knowledge, this is the first study that describes PREM and PROM items after hysterectomy in women with endometriosis and pelvic pain. The advantages of presenting PREMs and PROMs are apparent to healthcare professionals, as the measurements make it possible to examine and improve the healthcare in a patient-centered way, taking the needs and expectations of the patients into account.

Searching the literature, we found only two studies assessing self-reported patient satisfaction after hysterectomy ${ }^{22,23}$. Tay et al. ${ }^{18}$ in agreement with our results, found, in women with pelvic pain, a high satisfaction rate one year after the hysterectomy. Pitter et al. ${ }^{19}$ examined the impact of different surgical modalities on patient satisfaction and also found a high overall satisfaction rate after the hysterectomy but did not examine the associations with diagnosis or occurrence of pelvic pain.

An individual's interpretation of, response to and ability to cope with pain is dependent on emotional and cognitive factors such as current mental health status and history of trauma

${ }^{24}$. The high levels of anxiety, depression and other psychiatric disorders among women with painful endometriosis is well documented ${ }^{25-28}$. Due to limitations of the data in the GynOp we were not able to control for mental health status in our study. 
Hartmann et al. compared differences in quality of life and depression after hysterectomy. In that study, all women improved over the preoperative baseline, but women with both pelvic pain and depression fared less well than the others, and had the highest risk for poor outcomes in most categories. Besides, the women with "depression only" had the second highest risk, while the "pain only” group had just slightly higher odds for poor outcomes compared with the reference group with neither pain nor depression. This shows that depression might be the most important predictor for dissatisfaction, and pain can be interpreted as a contributing factor ${ }^{18}$. In our study, we found similar relations between endometriosis and pain: our results indicated that pelvic pain was the main factor for poor PREM and PROM ratings, and implied the occurrence of endometriosis diagnosis was a significant contributing factor. Berner et al. ${ }^{29}$ found significant improvement in pain and patient satisfaction one year after subtotal hysterectomy regardless of whether the women had endometriosis/adenomyosis or not.

Our results showed that women with pain and endometriosis experienced more complications, both during their hospital stay and up to one year postoperatively, than women without pelvic pain and endometriosis, which is in conformity with Uccella et al. ${ }^{30}$. One explanation may be that the surgery performed on women with pelvic pain and endometriosis was more complex and complicated (as indicated by the higher rate of "complications during hospital stay” in Table 2) and thus had higher risks of having late postoperative complications. Alternatively, the women might report dissatisfaction with the results of the surgery as late postoperative complications since these did not comply with their expectations, and consequently something associated with the surgery had gone wrong after the surgery.

Different types of endometriosis (peritoneal superficial endometriosis, deep infiltrating endometriosis and ovarian endometriomas) may cause different degrees of pain. The deep infiltrating endometriosis seems to cause more severe pain and has a 
higher recurrence rate than the other types of endometriosis ${ }^{31,32}$. It was not possible to evaluate the type of endometriosis in detail in the Gyn Op and consequently the interpretation of the results should be conducted with caution.

The study is based on a large body of prospectively collected material and standardized follow-up questionnaires from the GynOp, which strengthens the validity of the results. The study reflects the clinical situations leading to hysterectomy, covering a broad spectrum of different benign gynecological conditions and premenopausal women. Moreover, the response rates for both the eight-week and one-year follow-up questionnaires were high. All these factors increase the possibility of generalization of the results. The register has been the source for a number of previous studies and is considered to be well described and evaluated 33-36. However, as in all epidemiologic studies, the main limitation of this study is the risk of selection bias and lack of information, which may be potential confounders.

It may be unreliable to use endometriosis as the indication for the hysterectomy as a variable in the analyses, if not histopathologically proven preoperatively, since only $75 \%$ of women with histological confirmed endometriosis are symptomatic ${ }^{3}$. Very often, the indication for hysterectomy is based on symptom diagnoses such as menstrual bleeding disorder, physical discomfort due to fibroids or cysts, dysmenorrhea, dyspareunia or pain without any histopathological confirmation of the underlying condition. Consequently, we did not use indications for the hysterectomy in the analyses but used the final postoperative diagnosis after the hysterectomy as a measure of the occurrence of endometriosis in order to assess the associations between pelvic pain and endometriosis. Another reason for not including the indication is the focus on the patient's perspective in this study. Instead of using the doctors' indication, we based our index and comparison groups on the women's complaint of pelvic pain preoperatively. 
Although the overall response rate was high, the answering rate regarding certain PREM and PROM questions was lower. This was mainly attributed to changes in the construction of the GynOp over time leading to withdrawal or inclusion of variables or questions in the questionnaire. Taking this into consideration, even those variables/questions that showed high missing data rates actually had a high response rate, corresponding to the others when reporting for the period in which the specific data was collected.

The results of the study demonstrate the need for an effective postoperative treatment of the women with endometriosis and CPP. Treatment with dienogest significantly decreases endometriosis-related pain, similar to gonadotropin-releasing hormone agonists (GnRHa), both as initial and postoperative therapy, without the negative side effect profile of $\mathrm{GnRHa}^{37}$. The observation of prolonged pain relief even after the return of normal menses in women with endometriosis suggests a possible sustained effect on endometrial lesions. Dienogest may therefore represent an effective long-term medical treatment option for women with endometriosis ${ }^{38}$. Whether this also can be applied after hysterectomy for endometriosis and CPP remains to be proven.

\section{Conclusion}

The results indicate that the vast majority of the women were satisfied with the hysterectomy they had undergone but women with preoperative pelvic pain and a confirmed diagnosis of endometriosis scored lower on PREMs and PROMs compared with women undergoing hysterectomy for other benign conditions. The strongest associations for "negative” outcomes in PREMs and PROMs were found in the comparisons between women with pelvic pain and endometriosis vs. women without pelvic pain or endometriosis. This may imply that pelvic pain per se is the main factor for the rating in the PREMs and PROMs, and the occurrence of endometriosis is a significant contributing factor. This conclusion is supported by two recent reviews, one showing the negative effect of endometriosis-related pelvic pain on social life 
and psychological health ${ }^{26,27}$, and one by Facchin et al. ${ }^{28}$ who concluded that pain-free women with endometriosis did not differ from healthy controls in quality of life and psychological health. Endometriosis is a multidimensional, complex disease that involves the interaction of physical, psychological and social factors, and the research on the topic is still scant. More research is warranted to assess PREMs and PROMs and their influence on medical and surgical treatments in endometriosis. 


\section{Acknowledgments:}

We acknowledge the Swedish National Quality Registry of Gynecological Surgery (GynOp),

The Swedish Government and the Swedish Association of Local Authorities and Regions, which all support GynOp.

\section{Author disclosure statement}

No competing financial interests exist. 


\section{References}

1. Sourial S, Tempest N, Hapangama DK. Theories on the pathogenesis of endometriosis. Int J Reprod Med. 2014;2014:179515. doi:10.1155/2014/179515.

2. Laganà AS, Triolo $\mathrm{O}$, Salmeri FM, et al. Natural killer T cell subsets in eutopic and ectopic endometrium: a fresh look to a busy corner. Arch Gynecol Obstet.

2016;293(5):941-949. doi:10.1007/s00404-015-4004-7.

3. Vetvicka V, Laganà AS, Salmeri FM, et al. Regulation of apoptotic pathways during endometriosis: from the molecular basis to the future perspectives. Arch Gynecol Obstet. 2016;294(5):897-904. doi:10.1007/s00404-016-4195-6.

4. Matsuzaki S, Darcha C. Involvement of the Wnt/ $\beta$-catenin signaling pathway in the cellular and molecular mechanisms of fibrosis in endometriosis. Trackman PG, ed. PLoS One. 2013;8(10):e76808. doi:10.1371/joumal.pone.0076808.

5. Dunselman G, Vermeulen N, Becker C, et al. ESHRE guideline: management of women with endometriosis. Hum Reprod. 2014;29(3):400-412.

doi:10.1093/hummep/det457.

6. Fuldeore MJ, Soliman AM. Prevalence and s s Symptomatic B B $\underline{b} u r d e n$ of $\underline{d}$ Điagnosed

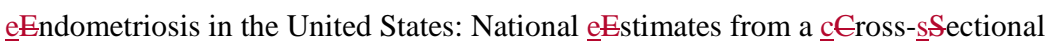
s Survey of 59,411 wWomen. Gynecol Obstet Invest. 2016;0. [Epub ahead of print]doi:10.1159/000452660.

7. Triolo O, Laganà AS, Sturlese E. Chronic pelvic pain in endometriosis: an overview. $J$ Clin Med Res. 2013;5(3):153-163. doi:10.4021/jocmr1288w.

8. Vercellini P, Somigliana E, Viganò P, Abbiati A, Barbara G, Fedele L. Chronic pelvic pain in women: etiology, pathogenesis and diagnostic approach. Gynecol Endocrinol. 2009;25(3):149-158. doi:10.1080/09513590802549858.

9. Romanek-Piva K, Gałczyński K, Adamiak-Godlewska A, Futyma K, Miotła P, 
Rechberger T. Hysterectomy trends for benign indications over a 15-year period in an academic teaching center in Poland: a retrospective cohort study. Ginekol Pol. 2016;87(6):411-416. doi:10.5603/GP.2016.0017.

10. Nanavati AM, Gokral SB. A p prospective rRandomized çGomparative s s Study of

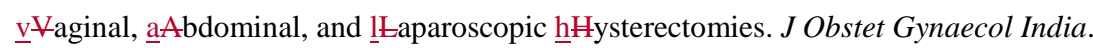
2016;66(Suppl 1):389-394. doi:10.1007/s13224-015-0756-z.

11. Parazzini F, Ricci E, Bulfoni G, et al. Hysterectomy rates for benign conditions are declining in Lombardy, Italy: 1996-2010. Eur J Obstet Gynecol Reprod Biol. 2014;178:107-113. doi:10.1016/j.ejogrb.2014.04.024.

12. Lundholm C, Forsgren C, Johansson AL V, Cnattingius S, Altman D. Hysterectomy on benign indications in Sweden 1987-2003: a nationwide trend analysis. Acta Obstet Gynecol Scand. 2009;88(1):52-58. doi:10.1080/00016340802596017.

13. Gorlero F, Lijoi D, Biamonti M, et al. Hysterectomy and women satisfaction: total versus subtotal technique. Arch Gynecol Obstet. 2008;278(5):405-410. doi:10.1007/s00404-008-0615-6.

14. Stovall TG, Ling FW, Crawford DA. Hysterectomy for chronic pelvic pain of presumed uterine etiology. Obstet Gynecol. 1990;75(4):676-679.

15. Hillis SD, Marchbanks PA, Peterson HB. The effectiveness of hysterectomy for chronic pelvic pain. Obstet Gynecol. 1995;86(6):941-945.

16. Brandsborg B, Nikolajsen L, Hansen CT, Kehlet H, Jensen TS. Risk factors for chronic pain after hysterectomy: a nationwide questionnaire and database study. Anesthesiology. 2007;106(5):1003-1012. doi:10.1097/01.anes.0000265161.39932.e8.

17. Vercellini P, Barbara G, Abbiati A, Somigliana E, Viganò P, Fedele L. Repetitive surgery for recurrent symptomatic endometriosis: what to do? Eur J Obstet Gynecol Reprod Biol. 2009;146(1):15-21. doi:10.1016/j.ejogrb.2009.05.007. 
18. Hartmann KE, Ma C, Lamvu GM, Langenberg PW, Steege JF, Kjerulff KH. Quality of life and sexual function after hysterectomy in women with preoperative pain and depression. Obstet Gynecol. 2004;104(4):701-709. doi:10.1097/01.AOG.0000140684.37428.48.

19. Rizk B, Fischer AS, Lotfy HA, et al. Recurrence of endometriosis after hysterectomy. Facts, views Vis ObGyn. 2014;6(4):219-227.

20. Nilsson E, Orwelius L, Kristenson M. Patient-reported outcomes in the Swedish National Quality Registers. J Intern Med. 2016;279(2):141-153. doi:10.1111/joim.12409.

21. Swedish Society of Obstetrics and Gynecology. The Swedish National Quality Register for Gynecological Surgery. [In Swedish]. http://www.gynop.org/english/about/about.htm. Published 2011. Accessed Octøber September 107, $201 \underline{76}$.

22. Tay SK, Bromwich N. Outcome of hysterectomy for pelvic pain in premenopausal women. Aust N Z J Obstet Gynaecol. 1998;38(1):72-76.

23. Pitter MC, Simmonds C, Seshadri-Kreaden U, Hubert HB. The $\underline{i} I m p a c t ~ o f ~ \underline{d} Đ$ ifferent $\underline{s}$ Surgical $\underline{m}$ Modalities for $\underline{h} H y$ sterectomy on $\underline{s}$ Satisfaction and $\underline{p} P a t i e n t \underline{r}$ Reported oӨutcomes. Interact J Med Res. 2014;3(3):e11. doi:10.2196/ijmr.3160.

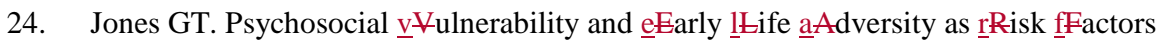

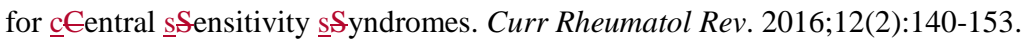

25. Laganà AS, La Rosa VL, Rapisarda AMC, et al. Anxiety and depression in patients with endometriosis: impact and management challenges. Int $J$ Womens Health. 2017;9:323-330. doi:10.2147/IJWH.S119729.

26. Pope CJ, Sharma V, Sharma S, Mazmanian D. A s s Systematic rReview of the

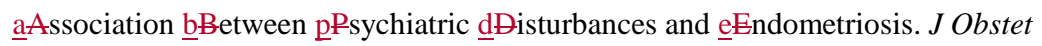


Gynaecol Canada. 2015;37(11):1006-1015. doi:10.1016/S1701-2163(16)30050-0.

27. Culley L, Law C, Hudson N, et al. The social and psychological impact of endometriosis on women's lives: a critical narrative review. Hum Reprod Update.

2013;19(6):625-639. doi:10.1093/humupd/dmt027.

28. Facchin F, Barbara G, Saita E, et al. Impact of endometriosis on quality of life and mental health: pelvic pain makes the difference. J Psychosom Obstet Gynecol.

2015;36(4):135-141. doi:10.3109/0167482X.2015.1074173.

29. Berner E, Qvigstad E, Myrvold AK, Lieng M. Pelvic pain and patient satisfaction after laparoscopic supracervical hysterectomy: prospective trial. J Minim Invasive Gynecol. 2014;21(3):406-411. doi:10.1016/j.jmig.2013.10.011.

30. Uccella S, Marconi N, Casarin J, et al. Impact of endometriosis on surgical outcomes and complications of total laparoscopic hysterectomy. Arch Gynecol Obstet.

2016;294(4):771-778. doi:10.1007/s00404-016-4115-9.

31. Sibiude J, Santulli P, Marcellin L, Borghese B, Dousset B, Chapron C. Association of

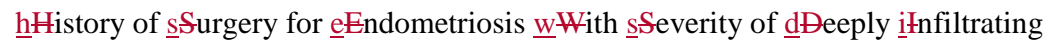
eEndometriosis. Obstet Gynecol. 2014;124(4):709-717. doi:10.1097/AOG.0000000000000464.

32. Laganà AS, Vitale SG, Trovato MA, et al. Full-Thickness ęExcision versus s shhaving

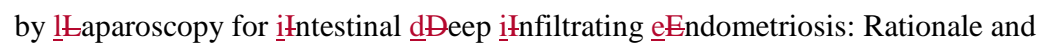
pPotential țTreatment ôPptions. Biomed Res Int. 2016;2016:3617179. doi:10.1155/2016/3617179.

33. Ladfors MB, Löfgren MEO, Gabriel B, Olsson J-HA. Patient accept questionnaires integrated in clinical routine: a study by the Swedish National Register for Gynecological Surgery. Acta Obstet Gynecol Scand. 2002;81(5):437-442.

34. Pakbaz M, Mogren I, Löfgren M. Outcomes of vaginal hysterectomy for uterovaginal 
prolapse: a population-based, retrospective, cross-sectional study of patient perceptions of results including sexual activity, urinary symptoms, and provided care. BMC Womens Health. 2009;9:9. doi:10.1186/1472-6874-9-9.

35. Nilsson M, Lalos O, Lindkvist H, Löfgren M, Lalos A. Female urinary incontinence: patient-reported outcomes 1 year after midurethral sling operations. Int Urogynecol J. 2012;23(10):1353-1359. doi:10.1007/s00192012 17529.

36. Nüssler E, Kesmodel US, Löfgren M, Nüssler EK. Operation for primary cystocele with anterior colporrhaphy or non-absorbable mesh: patient-reported outcomes. Int Urogynecol J. 2015;26(3):359-366. doi:10.1007/s00192 014 2511x.

37. Greene AD, Lang SA, Kendziorski JA, Sroga-Rios JM, Herzog TJ, Burns KA. Endometriosis: where are we and where are we going? Reproduction. 2016;152(3):R63-R78.doi:10.1530/REP 16-0052.

38. Petraglia F, Hornung D, Seitz C, et al. Reduced pelvic pain in women with endometriosis: efficacy of long-term dienogest treatment. Arch Gynecol Obstet. 2012;285(1):167-173. doi:10.1007/s00404-011-1941-7. 


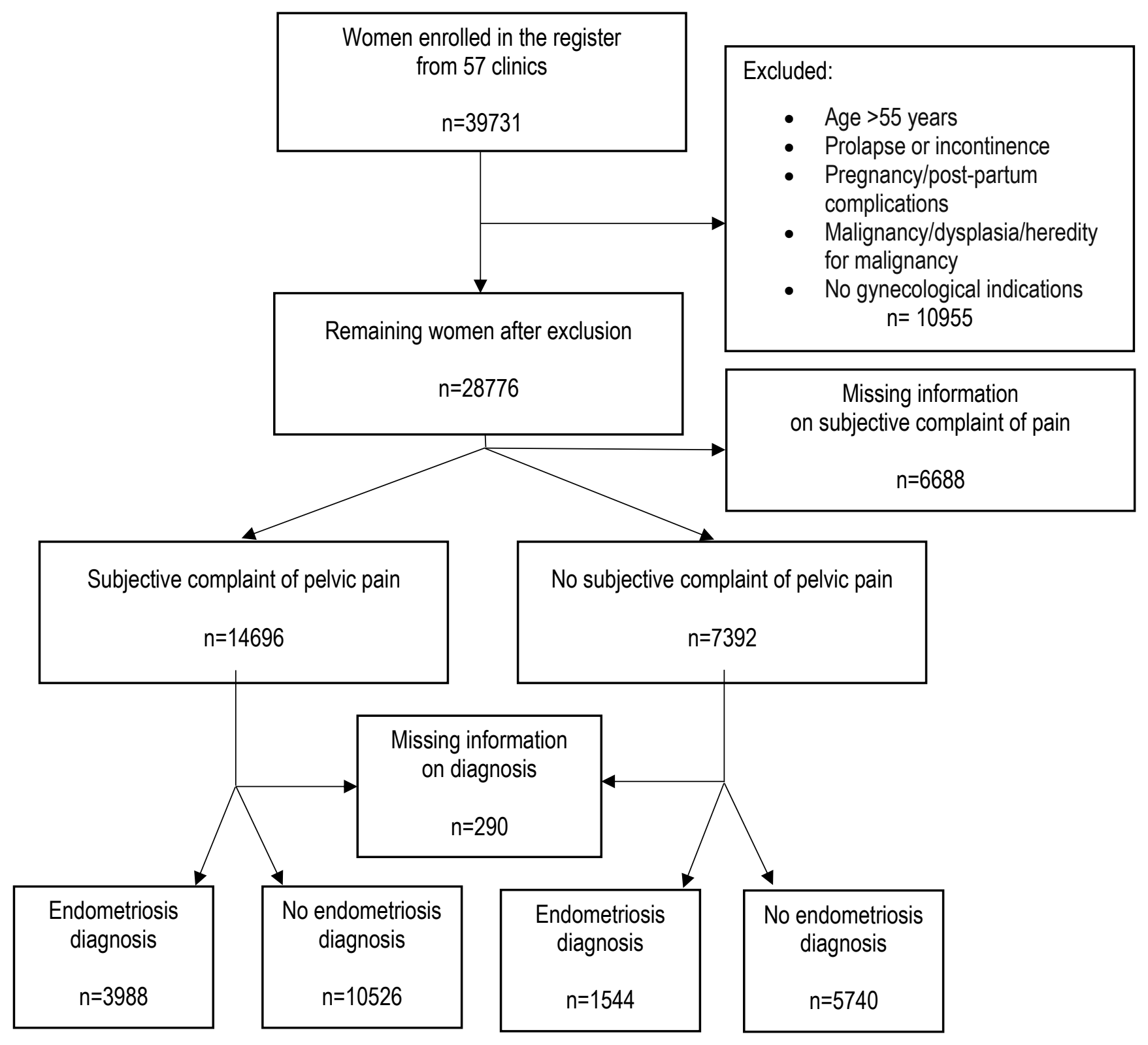


Title: Table 1 Demographic and descriptive data

Table 1. Demographic and descriptive data on participants subdivided after preoperative subjective complaint of pain and into index group (A) and comparison groups (B-D).

Subjective complaint of pain $\quad$ No subjective complaint of pain

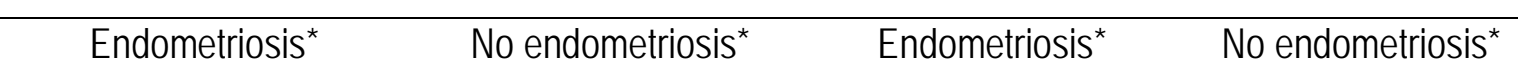

(A)

$(n=3988)$

$44.6(5.1 ; 25-55)$

120 (3.0)

$<35$ years

Age groups:

35-44 years

1687 (42.3)

45-55 years

Parity

BMI $\left(\mathrm{kg} / \mathrm{m}^{2}\right)$

BMI groups:

$\begin{array}{ll} & 2.2(1.2 ; 0-12) \\ & 26.7(4.8 ; 15.1-66.3) \\ <18.5 \mathrm{~kg} / \mathrm{m}^{2} & 26(0.6)\end{array}$

(B)

$$
\text { ( } n=10526)
$$
44.7 (5.2; 19-55)

397 (3.8)

4336 (41.2)

$5793(55.0)$

$2.1(1.2 ; 0-10)$

$26.3(4.7 ; 15.9-64.9)$

$98(1.0)$

(C)

(D)

$(n=5740)$

$(n=1544)$ $46.0(4.9 ; 21-55)$

114 (2.0)

24 (1.6)

1881 (32.8)

3745 (65.2)

$2.1(1.1 ; 0-10)$

$2.3(1.2 ; 0-9)$

$26.2(4.6 ; 16.1-59.9)$

$15(1.0)$

45 (0.8) 


$\begin{array}{llllll} & 18.5-24.9 \mathrm{~kg} / \mathrm{m}^{2} & 1606(42.0) & 4488(44.3) & 596(40.1) & 2576(46.4) \\ & 25.0-29.9 \mathrm{~kg} / \mathrm{m}^{2} & 1333(34.9) & 3528(34.8) & 534(35.9) & 1898(34.2) \\ & 30.0-34.9 \mathrm{~kg} / \mathrm{m}^{2} & 617(16.2) & 1498(14.8) & 243(16.4) & 766(13.8) \\ & \geq 35.0 \mathrm{~kg} / \mathrm{m}^{2} & 239(6.3) & 513(5.1) & 98(6.6) & 263(4.8) \\ \text { ASA classification: } & \text { ASA 1 } & 2895(74.4) & 8010(77.8) & 1155(76.4) & 4447(78.9) \\ & \text { ASA 2 } & 953(24.4) & 2180(21.2) & 340(22.5) & 1128(20.0) \\ \text { Smoking } & \text { ASA 3 } & 45(1.2) & 101(1.0) & 14(0.91) & 60(1.1) \\ & & 824(20.9) & 2144(20.7) & 267(17.6) & 827(14.6) \\ \text { Employment } & \text { Full time } & 1467(49.7) & 4079(51.9) & 686(59.4) & 2616(61.0) \\ & \text { Part time } & 616(20.9) & 1648(21.0) & 243(21.0) & 898(20.9) \\ & \text { Not working\# } & 868(29.4) & 2127(27.1) & 226(19.6) & 777(18.1)\end{array}$

Figures denote mean and (SD; range) or number of women and (per cent). ASA = American Society of Anesthesiologists; BMI = body mass index; $\mathrm{SD}=$ Standard Deviation

*Diagnosis confirmed histopathologically. *On sick leave/maternity leave/disability pension/unemployed. 

Title: Table 2. Clinical data

Table 2. Clinical data of participants subdivided after preoperative subjective complaint of pain and into index group (A) and comparison groups (B-D).

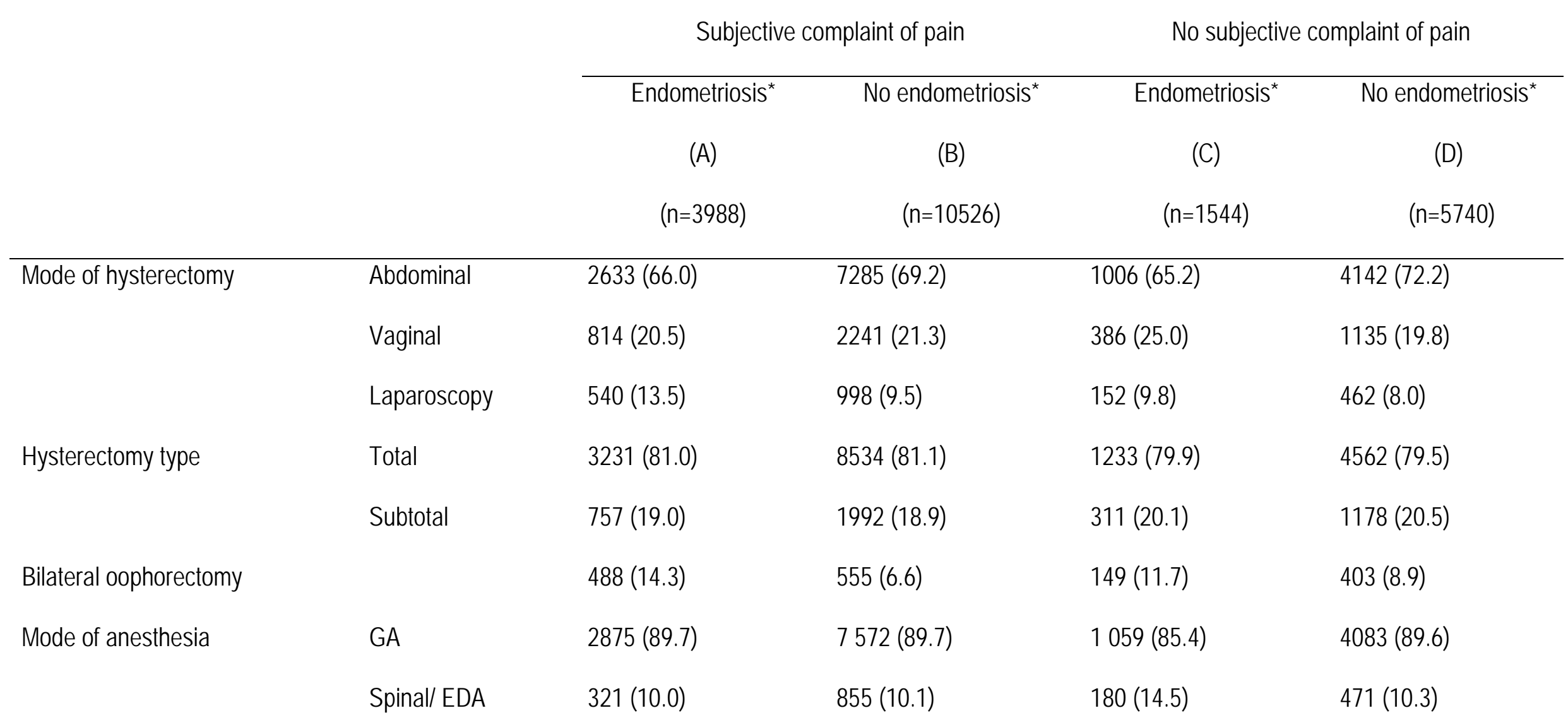


Figures denote number of women and (per cent). EDA, epidural analgesia; GA, general anesthesia; LA, local anesthesia.

*Diagnosis confirmed histopathologically. 

Title: Table 3. PREM and PROM questions.

Table 3. The PREM and PROM questions from the eight-week and one-year questionnaires and the prevalence of the dichotomized answers.

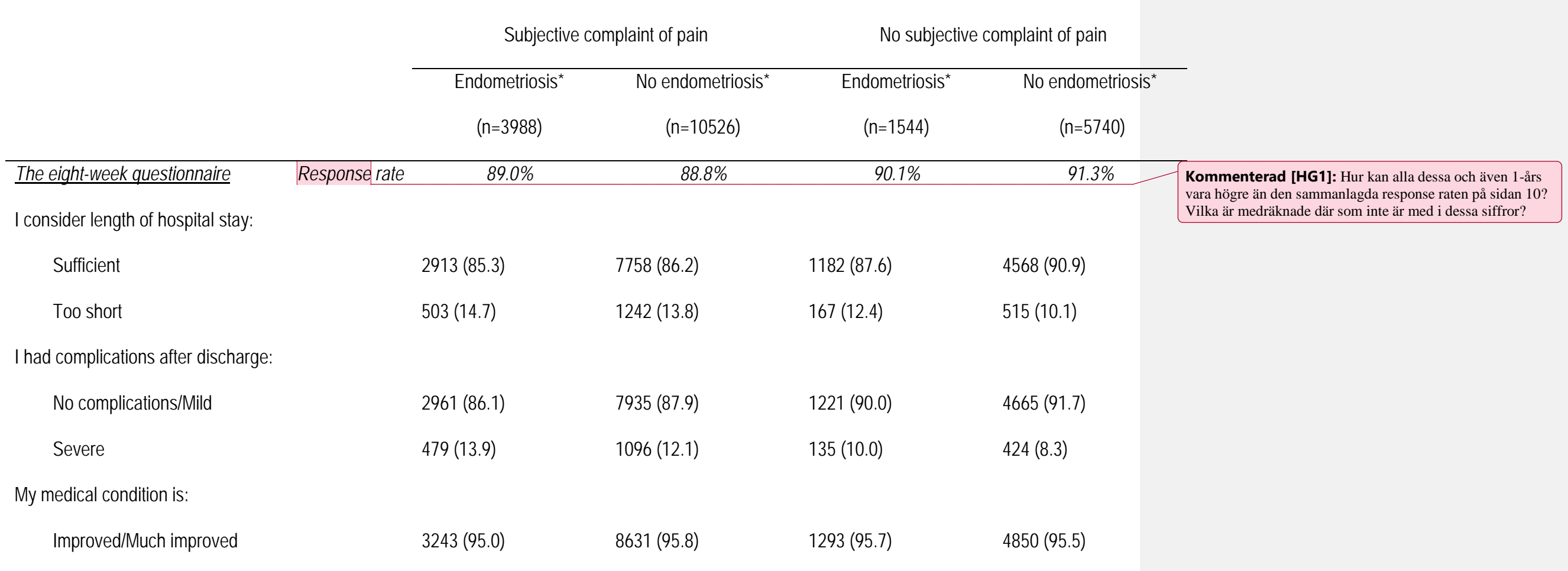


Regarding the result of the operation, I am:

\begin{tabular}{|c|c|c|c|c|}
\hline Satisfied/Very satisfied & $2695(91.3)$ & $7304(92.9)$ & $1158(94.7)$ & $4345(95.4)$ \\
\hline Neither-nor/Dissatisfied/Very dissatisfied & $256(8.7)$ & $556(7.1)$ & $65(5.3)$ & $212(4.6)$ \\
\hline No complications/Mild & $2794(93.9)$ & $7530(95.1)$ & $1188(96.6)$ & $4429(97.4)$ \\
\hline Severe & $182(6.1)$ & $385(4.9)$ & $42(3.4)$ & $119(2.6)$ \\
\hline Improved/Much improved & 1979 (95.2) & 5088 (95.7) & $859(95.9)$ & $3155(95.7)$ \\
\hline Unchanged/Worse/Much worse & $99(4.8)$ & $228(4.3)$ & $37(4.1)$ & $141(4.3)$ \\
\hline
\end{tabular}

Figures denote number of women and (per cent of responders).

*Diagnosis confirmed histopathologically. 


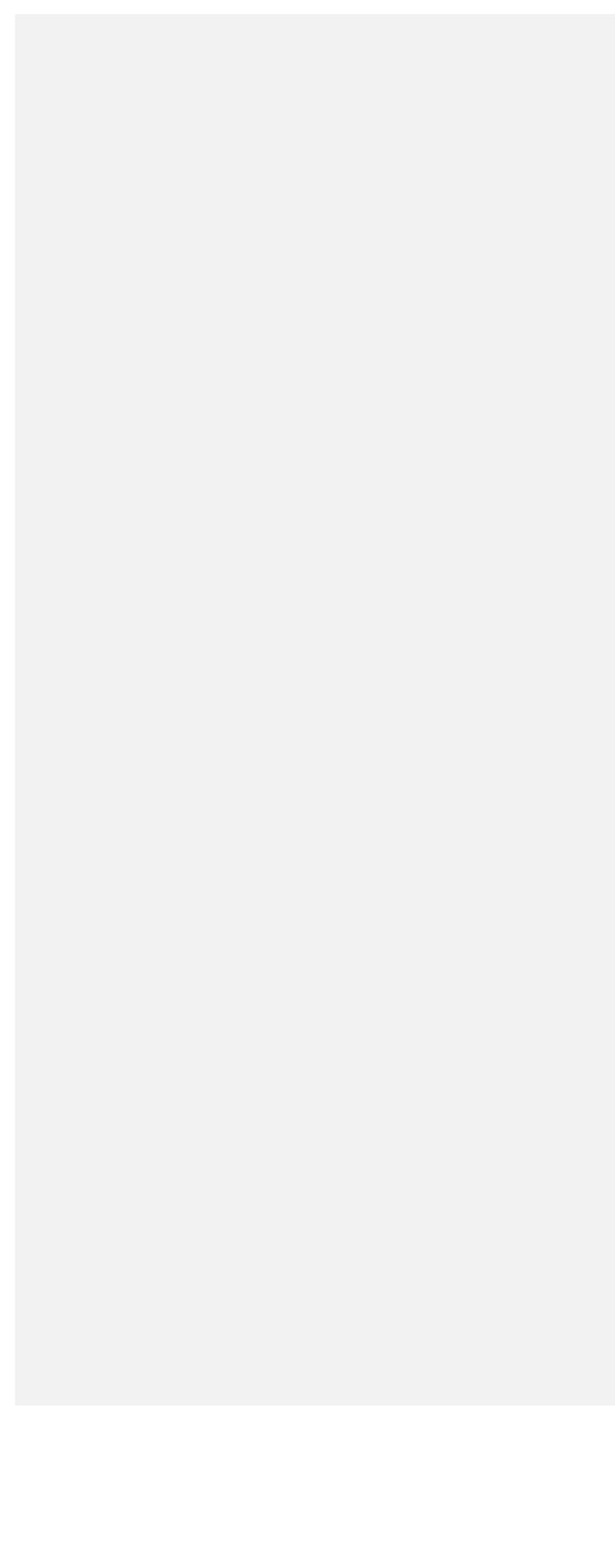


Title: Table 4. Results of the logistic regression analyses.

Table 4. Results of the logistic regression analyses of the comparisons between the groups concerning the PREM and PROM questions in the eight-week and one-year questionnaires.

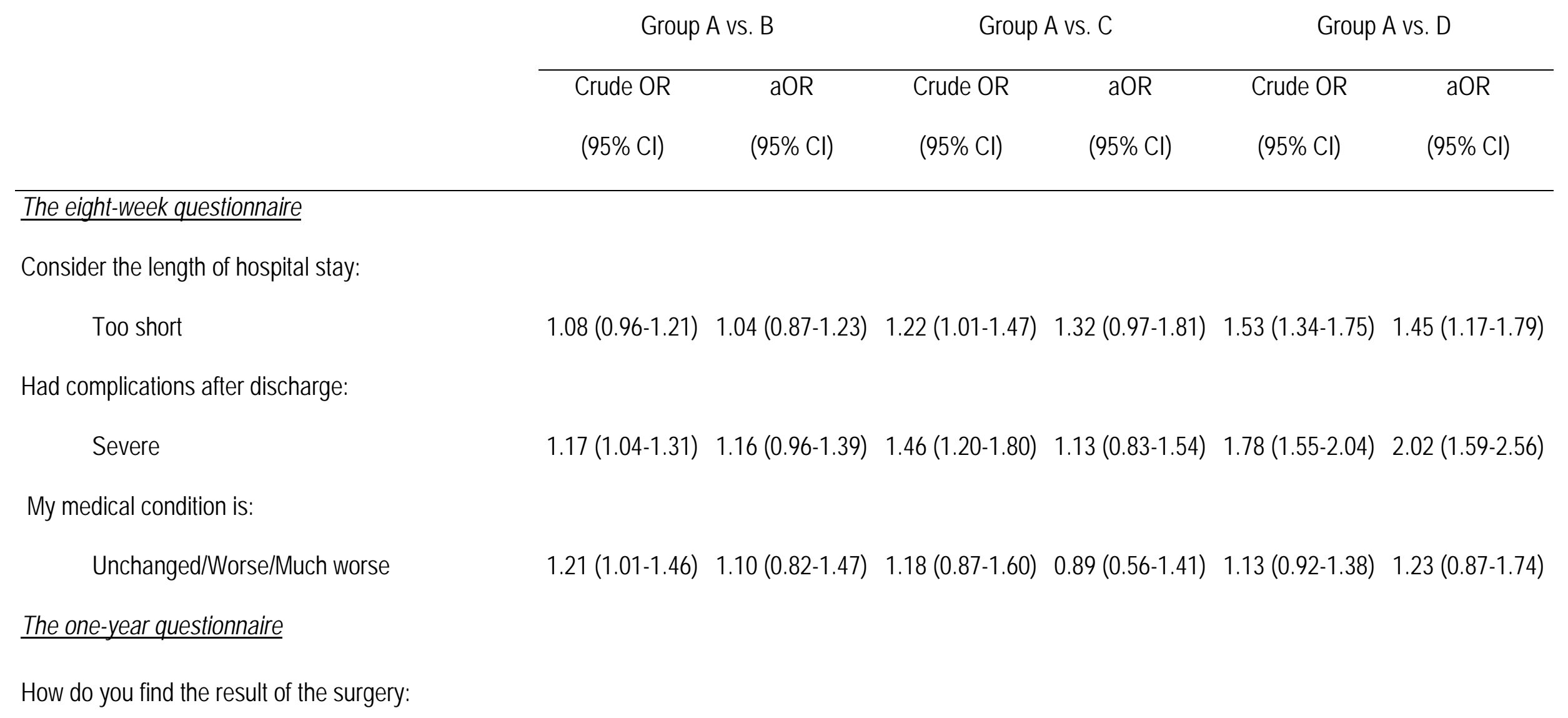


Had complications after discharge:

Severe

$1.27(1.06-1.53) \quad 1.37(1.03-1.82) \quad 1.84(1.31-2.59) \quad 1.42(0.85-2.37) \quad 2.42(1.92-3.07) \quad 2.31(1.57-3.39)$

My medical condition is:

Unchanged/Worse/Much worse

$1.12(0.88-1.42) \quad 1.03(0.71-1.50) \quad 1.16(0.79-1.71) \quad 0.98(0.54-1.78) \quad 1.12(0.86-1.46) \quad 0.86(0.57-1.30)$

aOR, adjusted odds ratio; CI, confidence interval; OR, odds ratio.

In the multivariate logistic regression models, adjustment was made for age, parity, BMI, ASA classification, smoking habits, employment,

mode of hysterectomy, hysterectomy type, occurrence of bilateral oophorectomy, mode of anesthesia and occurrence of complications

during hospital stay. 